\title{
Application of the Improved Grey Model in the Monthly Electricity Consumption Forecasting
}

\author{
Zhang Wenzhe ${ }^{1}$, Li Yangyang ${ }^{2}$ \\ ${ }^{1}$ State Grid Chong Qing Electric Power Company, Chongqing, China \\ ${ }^{2}$ Central China Science and Technology Development Co., Ltd., Wuhan, China
}

Email address:

375235250@qq.com (Zhang Wenzhe), liyy@sgepit.com (Li Yangyang)

\section{To cite this article:}

Zhang Wenzhe, Li Yangyang. Application of the Improved Grey Model in the Monthly Electricity Consumption Forecasting. Science Discovery. Vol. 4, No. 1, 2016, pp. 1-5. doi: 10.11648/j.sd.20160401.11

Received: January 26, 2016; Accepted: March 11, 2016; Published: April 8, 2016

\begin{abstract}
The paper improves the prediction accuracy of the monthly electricity consumption of power system, a hybrid prediction model is put forward aiming at the problems existing in the traditional grey prediction method, which is based on the combined optimization model of particle swarm and $\mathrm{K}$ nearest value. Grey prediction equation is solved by particle swarm optimization algorithm, which is a good solution to the problem of the choice of parameters of gray prediction equation, with strong global optimization ability; A combinatorial optimization algorithm of the K- nearest value and particle swarm was proposed, which solves the problem of prediction error caused by large fluctuations of raw data, and improves the accuracy of prediction results. Through the prediction of monthly electricity consumption in the past years, the results show that the combination prediction method proposed in this paper can effectively predict the monthly electricity consumption, and is practical.
\end{abstract}

Keywords: Monthly Prediction of Electricity Consumption, Particle Swarm, K Nearest Neighbor, Combination Optimization

\section{改进灰色模型在月度用电量预测中的应用}

\author{
张文哲 ${ }^{1}$, 李阳阳 ${ }^{2}$ \\ ${ }^{1}$ 国网重庆市电力公司, 重庆市, 中国 \\ ${ }^{2}$ 湖北国网华中科技开发有限责任公司, 湖北省武汉市, 中国 \\ 邮箱 \\ 375235250@qq. com（张文哲），1iyy@sgepit.com（李阳阳）
}

摘要: 为了提高电力系统月度用电量的预测精度, 本文针对传统灰色预测方法中存在的问题, 提出一种混合预测模型: 基于粒子群- $\mathrm{K}$ 最邻近值组合优化的改进灰色模型。通过粒子群算法对灰色预测方程进行求解, 可以很好的解决灰色预 测方程参数的选择问题, 具有很强的全局寻优能力; 提出的粒子群-K最邻近值组合优化算法, 解决了因原始数据波动 较大而导致的预测误差偏大问题, 提高了预测结果的准确性。通过对历年月度用电量的预测, 结果表明本文所提出的 组合预测方法可以有效的预测月度用电量, 具有一定的实用性。

关键词: 月度用电量预测, 粒子群, K最邻近, 组合优化 


\section{1. 引言}

中长期的用电量预测是电力系统的重要工作之一, 既 是合理制定购电计划的前提, 也是安排调度电力系统安全 运行的重要保障。依靠准确的电量预测, 可以合理地安排 电网内部发电机组的启停, 保持电网运行的稳定性, 合理 安排机组检修, 保证社会的正常生产和人民群众的正常生 活, 有效降低发电成本, 提高经济效益和社会效益。由于 影响电力预测的因素很多, 为了提高预测的准确度, 大量 学者对此进行了研究, 主要分为两大类: 一是参数估计的 预测方法, 主要包括回归分析法 [1], 灰色预测法 [2], 时 间序列法 [3], 相关分析法 [4] 等; 二是人工智能方法, 主 要包括支持向量机 [5], 神经网络预测 [6], 模拟退火法 [7], 数据挖掘技术 $[8]$ 等智能预测方法。

灰色预测方法是一种对含有不确定因素的系统进行 预测的方法。Morita Hironobu等人在1995年首次将灰色 系统理论应用于电力负荷预测, 将其作为一种新的负荷 预测方法 [9]; Hsu CC等人提出一种改进的GM模型, 将其 应用于台湾电力负荷预测 [10]; 文献 [11] 中通过对灰色 模型进行改进, 建立了反馈修正模型用于中长期负荷预 测；文献 [12]通过不同的权重确定方法，建立了灰色最 优组合模型，对中长期负荷进行预测；不少学者还将灰 色预测方法与其他方法相结合, 来提高预测精度, 如灰 色一支持向量机 [13], 灰色-神经网络组合 [14], 灰色一 回归分析 [15]等等。虽然经过长期的研究, 灰色预测方 法有了很大的发展, 取得了较好的成果, 但是其中仍存 在一些问题没能得到解决, 如: 模型边值的选择 [17], 模型参数的优化 $[18]$ 等。

本文基于月度统调用电量的年度发展序列, 首先通过 紧邻值法对原始数据进行光滑处理, 提出基于灰色-粒子 群算法来解决灰色预测模型中的参数计算问题, 通过与 $\mathrm{K}$ 最邻近算法组合优化, 解决因原始数据波动较大而导致的 预测偏差, 并通过实例证明本文所提方法的正确性及有效 性, 可将其用于对月度用电量的预测。

\section{2. 预测方法的原理}

\section{1. 原始数据的预处理}

针对原始数据中存在的异常波动采用紧邻值法进行 修正, 修正公式为:

$$
x^{\prime}(k)=\frac{x(k-1)+2 x(k)+x(k+1)}{4}
$$

对于数据起点, 有:

$$
x^{\prime}(1)=\frac{3 x(1)+x(2)}{4}
$$

对于数据终点, 有:

$$
x^{\prime}(n)=\frac{3 x(n)+x(n-1)}{4}
$$

式中, $k$ 为异常点的序列号, $\mathrm{k} \in(1, \ldots, \mathrm{n}), n$ 为序列 长度。

\section{2. 灰色GM $(1,1)$ 模型 $[16]$}

定义: $\mathrm{X}^{(0)}=\left(\mathrm{x}^{(0)}(1), \cdots, \mathrm{X}^{(0)}(\mathrm{n})\right)$ 为原始数据序列; $\mathrm{X}^{(1)}=\left(\mathrm{x}^{(1)}(1), \cdots, \mathrm{x}^{(1)}(\mathrm{n})\right)$ 为 $\mathrm{X}^{(0)}$ 的一阶累加生成序列; $Z^{(1)}=\left(z^{(1)}(2), \cdots, z^{(1)}(n)\right)$ 为 $X^{(1)}$ 的邻近均值生成序列, 其中, $\mathrm{z}^{(1)}(\mathrm{k})=\frac{1}{2}\left[\mathrm{x}^{(1)}(\mathrm{k})+\mathrm{x}^{(1)}(\mathrm{k}-1)\right], k=2,3, \cdots, n$; 称:

$$
\mathrm{x}^{(0)}(\mathrm{k})+\mathrm{az} \mathrm{z}^{(1)}(\mathrm{k})=\mathrm{b}
$$

为 GM $(1,1)$ 模型的基本形式。

设 $\mathrm{A}=[\mathrm{a}, \mathrm{b}]^{\mathrm{T}}$ 为参数列, $\mathrm{Y}=\left[\mathrm{x}^{(0)}(2), \cdots, \mathrm{x}^{(0)}(\mathrm{n})\right]^{\mathrm{T}}, \mathrm{B}=$ $\left[\begin{array}{cc}-\mathrm{z}^{(1)}(2) & 1 \\ \vdots & \vdots \\ -\mathrm{z}^{(1)}(\mathrm{n}) & 1\end{array}\right]$, 则 $\mathrm{GM}(1,1)$ 模型 $\mathrm{x}^{(0)}(\mathrm{k})+\mathrm{az}(\mathrm{l})=\mathrm{b}$ 的最 小二乘估计参数列满足下式:

$$
A=\left(B^{T} B\right)^{-1} B^{T} Y
$$

从而有GM $(1,1)$ 的白化微分方程:

$$
\frac{\mathrm{dx}^{(1)}}{\mathrm{dt}}+\mathrm{ax}^{(1)}=\mathrm{b}
$$

对上式求解可得

$$
\begin{aligned}
x^{(1)}(k+1) & =\left(C-\frac{b}{a}\right) e^{-a k}+\frac{b}{a} \\
x^{(0)}(k+1) & =x^{(1)}(k+1)-x^{(1)}(k) \\
& =\left(1-e^{a}\right)\left(C-\frac{b}{a}\right) e^{-a k}
\end{aligned}
$$

式中: $C$ 为特定的常数, 选择不同的初始条件, 可得 到不同的值。常数 $C$ 的求解表达式如下:

$$
\mathrm{x}^{(0)}(\mathrm{m})=\left(1-\mathrm{e}^{\mathrm{a}}\right)\left(\mathrm{C}-\frac{\mathrm{b}}{\mathrm{a}}\right) \mathrm{e}^{-(\mathrm{m}-1) \mathrm{a}}
$$

可得：

$$
\mathrm{C}=\frac{\mathrm{x}^{(0)}(\mathrm{m}) \mathrm{e}^{(\mathrm{m}-1) \mathrm{a}}}{1-\mathrm{e}^{\mathrm{a}}}+\frac{\mathrm{b}}{\mathrm{a}}
$$

式中: $m$ 为确定常数 $C$ 时的初始时刻, $m \in(1, \ldots, \mathrm{n})$ 根据式 (8) 和 (10) 可得灰色预测方程如下: 


$$
\left\{\begin{array}{c}
\mathrm{x}^{(0)}(\mathrm{k}+1)=\left(1-\mathrm{e}^{\mathrm{a}}\right)\left(\mathrm{C}-\frac{\mathrm{b}}{\mathrm{a}}\right) \mathrm{e}^{-\mathrm{ak}} \\
\mathrm{C}=\frac{\mathrm{x}^{(0)}(\mathrm{m}) \mathrm{e}^{(\mathrm{m}-1) \mathrm{a}}}{1-\mathrm{e}^{\mathrm{a}}}+\frac{\mathrm{b}}{\mathrm{a}}
\end{array}\right.
$$

\section{3. 对灰色预测模型的改进}

\section{1. 粒子群算法对参数求解}

在上述灰色预测算法中, 参数 $a, b$ 与背景值 $Z^{(1)}$ 的设 置紧密相关, 简单设置 $Z^{(1)}$ 的值容易影响 $a, b$ 的准确性, 产生误差; 另外不同初始时刻下常数 $C$ 的值不同，通过主 观判断设置的初始时刻极易导致方程（11）结果产生较大 误差。

粒子群算法从随机解出发, 通过迭代寻找 $a, b$ 的最优 解, 具体步骤如下:

(1) 对 $(a, b)$ 进行初始化, 将 $(a, b)$ 作为二维搜 索空间中的一个微粒;

（2）选择 $w$ 个粒子，每个粒子表示为一个二维向量, 用 $S$ 表示。 $\mathrm{S}_{\mathrm{i}}=\left(\mathrm{x}_{\mathrm{i}}, \mathrm{y}_{\mathrm{i}}\right) ， \mathrm{i}=1,2, \cdots, w$ 则第 $i$ 个粒 子的位置即为方程 (11) 的一个潜在解;

（3）比较每个粒子当前性能与有过的最优性能, 记第 $i$ 个粒子迄今为止搜索到的最优位置为 $P_{\mathrm{i}}=\left(P_{\mathrm{i} 1}\right.$, $\left.P_{\mathrm{i} 2}\right)$; 整个粒子群搜索到的最优位置则为 $P_{\mathrm{g}}=\left(P_{\mathrm{g} 1}, P_{\mathrm{g} 2}\right)$;

（4）改进粒子迭代速度:

$$
\mathrm{V}_{\mathrm{i}}^{\mathrm{t}+1}=\mathrm{V}_{\mathrm{i}}^{\mathrm{t}}+\mathrm{c}_{1} \mathrm{r}_{1}\left(\mathrm{P}_{\mathrm{i}}-\mathrm{S}_{\mathrm{i}}^{\mathrm{t}}\right)+\mathrm{c}_{2} \mathrm{r}_{2}\left(\mathrm{P}_{\mathrm{g}}-\mathrm{S}_{\mathrm{i}}^{\mathrm{t}}\right)
$$

式中： $i$ 表示第 $i$ 个粒子, $t$ 为迭代次数, $V_{i}$ 为一个二 维向量, 表示粒子迭代速度, 学习因子 $c_{1}$ 和 $c_{2}$ 是非负常数, $r_{1}$ 和 $r_{2}$ 为伪随机数, 服从 $[0,1]$ 均匀分布; $\mathrm{V}_{\mathrm{i}}^{1} \in[-\mathrm{v}, \mathrm{v}]$, $v$ 由用户设定。

(5) 改变第 $i$ 个粒子的位置:

$$
\mathrm{S}_{\mathrm{i}}^{\mathrm{t}+1}=\mathrm{S}_{\mathrm{i}}^{\mathrm{t}}+\mathrm{V}_{\mathrm{i}}^{\mathrm{t}}
$$

当迭代次数大于最大迭代次数或满足收玫条件, 则结 束迭代并输出结果, 否则转至 (3) 继续迭代。

\section{2. K最邻近算法的基本原理}

虽然基于粒子群算法对灰色预测模型加以改进，提高 了预测的精确度, 但是电力预测严重依赖原始数据, 用电 量数据出现较大波动将影响预测的准确度。本文采用 $\mathrm{K}$ 最 邻近算法对后验差检验不合格的序列进行预测, 经过与粒 子群算法组合优化使预测精度有进一步提高。

$\mathrm{K}$ 最邻近算法的主要思想是从一个特征空间中找到一 个样本的 $k$ 个最近邻居, 将不同邻居对该样本产生的影响 给予不同的权值。

设任意 1 个实例, 用 $X=\left\{t_{1}, t_{2}, \cdots, t_{n}\right\}$ 描述，两个实例 $X_{1}$ 和 $X_{2}$ 的距离通过下式求得:

$$
\mathrm{d}\left(\mathrm{X}_{1}, \mathrm{X}_{2}\right)=\sqrt{\sum_{\mathrm{i}=1}^{\mathrm{n}}\left(\mathrm{t}_{\mathrm{i}}-\mathrm{t}_{\mathrm{j}}\right)^{2}}
$$

建立预测公式:

$$
f^{\prime}(x)=\omega_{0}+\omega_{1} a_{1}(x)+\cdots+\omega_{n} a_{n}(x)
$$

式中 $\omega_{i}$ 为第 $i$ 个邻居的权重值, 由下式得到:

$$
\omega_{\mathrm{i}}=\frac{1}{\mathrm{~d}\left(\mathrm{x}, \mathrm{x}_{\mathrm{i}}\right)^{2}}
$$

式中: $x$ 为预测点, $x i$ 为 $x$ 的邻近点, 两者之间距离的 倒数为权重值。

在式 (15) 中, $\omega_{0}$ 为常数项, $\mathrm{f}^{\prime}(\mathrm{x})$ 为预测值, $\mathrm{a}_{\mathrm{i}}(\mathrm{x})$ 为第 $\mathrm{i}$ 个邻居的属性值。

\section{3. 组合优化方法}

假设 $f_{1}$ 是通过灰色一粒子群算法得到的预测值, $f_{2}$ 是通 过 $K$ 邻近算法得到的预测值, $f_{c}$ 为通过两种方法加权平均 得到的组合预测结果。假设这两种方法单独预测的误差为 $e_{1}, e_{2}$, 组合预测的误差为 $e_{c}, \omega_{1}^{\prime}, \omega_{2}^{\prime}$ 分别为两种方法预 测值的权重, 则有 $\omega_{1}^{\prime}+\omega_{2}^{\prime}=1, \mathrm{f}_{\mathrm{c}}=\omega_{1}^{\prime} \mathrm{f}_{1}+\omega_{2}^{\prime} \mathrm{f}_{2}$ 。

组合预测值的误差及方差则为:

$$
\begin{gathered}
\mathrm{e}_{\mathrm{c}}=\omega_{1}^{\prime} \mathrm{e}_{1}+\omega_{2}^{\prime} \mathrm{e}_{2} \\
\operatorname{var}\left(\mathrm{e}_{\mathrm{c}}\right)=\omega_{1}^{\prime 2} \operatorname{var}\left(\mathrm{e}_{1}\right)+\omega_{2}^{\prime 2} \operatorname{var}\left(\mathrm{e}_{2}\right)+2 \omega_{1}^{\prime} \omega_{2}^{\prime} \operatorname{cov}\left(\mathrm{e}_{1}, \mathrm{e}_{2}\right)
\end{gathered}
$$

基于两种方法是相互独立的, 因此有

$$
\omega_{1}^{\prime}=\frac{\operatorname{var}\left(\mathrm{e}_{2}\right)}{\operatorname{var}\left(\mathrm{e}_{1}\right)+\operatorname{var}\left(\mathrm{e}_{2}\right)}, \quad \omega_{2}^{\prime}=\frac{\operatorname{var}\left(\mathrm{e}_{1}\right)}{\operatorname{var}\left(\mathrm{e}_{1}\right)+\operatorname{var}\left(\mathrm{e}_{2}\right)}
$$

\section{4. 后验差检验}

本文采取的后验差检验指标如下所示:

设 $f(x)$ 和 $f^{\prime}(x)$ 分别为第 $i$ 月的实际用电量和预测值, 则有:

平均相对误差:

$$
E_{1}=\frac{1}{n} \sum_{i=1}^{n}\left|\frac{f(x)-f^{\prime}(x)}{f(x)}\right| \times 100 \%
$$

其中, $E_{1}$ 为序列平均相对误差。

均方根误差:

$$
E_{2}=\sqrt{\frac{1}{n} \sum_{i=1}^{n}\left|\frac{f(x)-f^{\prime}(x)}{f(x)}\right|^{2}} \times 100 \%
$$




\section{5. 本文算法程序流程图}

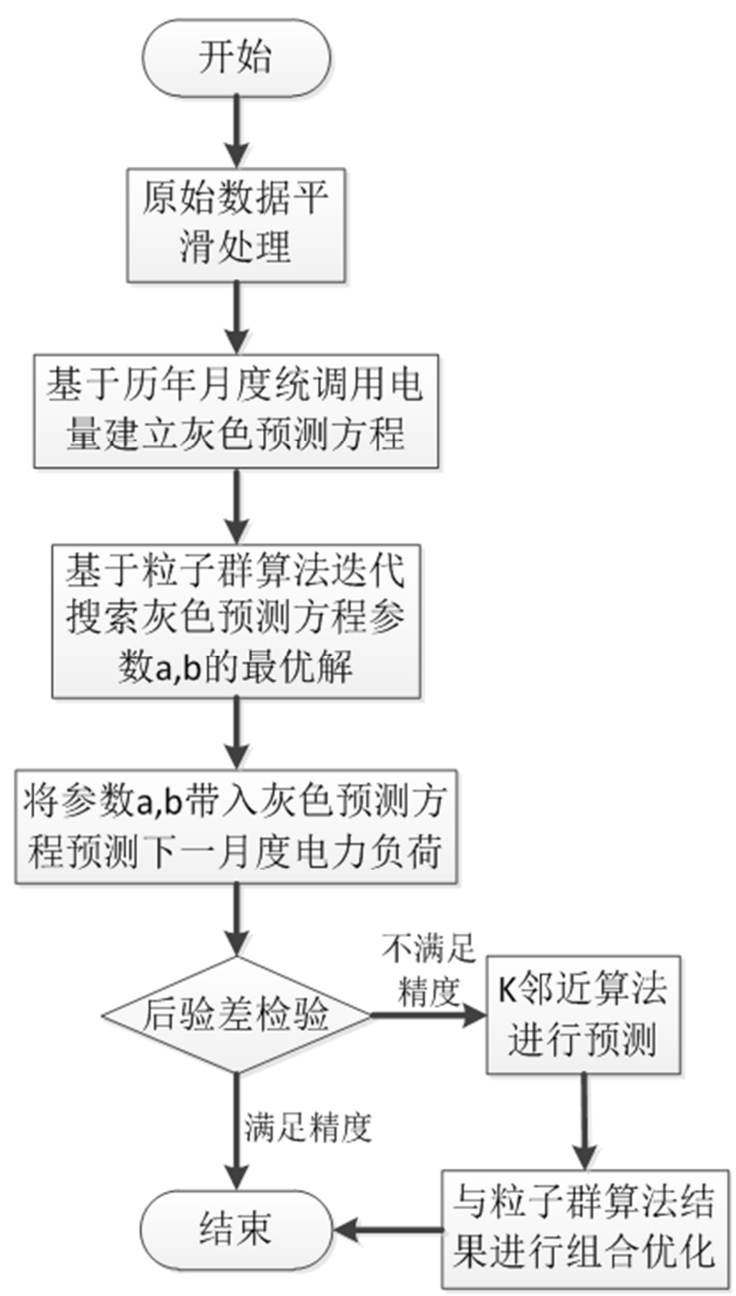

图1 算法流程图。

\section{4. 实例验证}

下面结合实际的数据讨论上述方法的实施过程。本文 选取国内某地区2008年至2013年的月度统调用电量数据, 进行仿真计算, 来验证方法的正确性。

以3、5、8、11四个月的2008-2012年月度统调用电量 数据为基础, 以 2013 年的月度统调用电量为预测目标, 建 立灰色一粒子群预测模型。

(1) 首先对原始数据进行预处理, 避免波动较大数 据对预测结果的影响; 表1为经过平滑处理后的国内某地 区历年月度统调用电量。

表1 数据平滑处理 (亿千瓦时)。

\begin{tabular}{lllll}
\hline 年\月 & $\mathbf{3}$ & $\mathbf{5}$ & $\mathbf{8}$ & $\mathbf{1 1}$ \\
\hline 2008 & 434 & 429 & 481 & 374 \\
2009 & 442 & 448 & 539 & 525 \\
2010 & 565 & 520 & 636 & 570 \\
2011 & 651 & 590 & 723 & 599 \\
2012 & 681 & 597 & 716 & 634 \\
2013 & 669 & 634 & 875 & 681 \\
\hline
\end{tabular}

以 11 月份数据为例, 根据 1.2 中定义计算式 (5), 获 得参数 $a, b$ 的初值, 得 $a=0.061, b=491$; 设置 $m$, 选择不 同的初始时刻带入式 (11)，选择合适的C值。表3中列出 了 $\mathrm{m}=1, \cdots, 5$ 时, 采用灰色预测模型对 2013 年11月份电力 用电量的预测结果。

表2 $\mathrm{m}$ 值对两种方法预测值的影响。

\begin{tabular}{lllll}
\hline & \multicolumn{2}{l}{ 灰色预测方法 } & \multicolumn{2}{l}{ 灰色-粒子群 } \\
\hline $\mathrm{m}$ 值 & 预测值 & 相对误差 (\%) & 预测值 & 相对误差 $(\%)$ \\
\hline 1 & 374 & 45.1 & 684.98 & 0.58 \\
2 & 525 & 22.9 & 683.15 & 0.32 \\
3 & 570 & 16.3 & 687.66 & 0.98 \\
4 & 599 & 12.0 & 683.40 & 0.35 \\
5 & 634 & 6.9 & 685.89 & 0.72 \\
\hline
\end{tabular}

由表2可知, 方程 (11) 中初值的选择对预测结果有 很大的影响, 所选时刻越接近预测时间越精确, 但计算结 果相对误差远大于灰色一粒子群算法。

（3）通过粒子群算法对方程（11）中的参数a, b进 行迭代搜索, 获取最优值, 可以避免选择不同的 $\mathrm{C}$ 值导致 的算法误差。

以2008年至 2012 年数据预测 2013 年月度统调用电量, 结果如下表所示:

表3 对2013年不同月份用电量的预测。

\begin{tabular}{|c|c|c|c|c|c|}
\hline 月份 & & 3 & 5 & 8 & 11 \\
\hline 预测值 & & 767 & 649 & 859 & 685.9 \\
\hline 相对误差 (\%) & & 14.65 & 2.37 & 1.83 & 0.72 \\
\hline \multirow{2}{*}{ 参数值 } & $\mathrm{a}$ & -0.114 & -0.083 & -0.116 & -0.068 \\
\hline & $\mathrm{b}$ & 383 & 449 & 394 & 486 \\
\hline
\end{tabular}

对预测方法进行后验差检验, 有:

表4 灰色-粒子群算法的后验差检验结果。

\begin{tabular}{lllll}
\hline 月份 & $\mathbf{3}$ & $\mathbf{5}$ & $\mathbf{8}$ & $\mathbf{1 1}$ \\
\hline 平均相对误差 (\%) & 6.98 & 3.2 & 4.0 & 0.83 \\
均方根误差 (\%) & 8.55 & 3.86 & 4.68 & 1.04 \\
\hline
\end{tabular}

从表3和表 4 可以看出，5、8、11月所得预测结果相对 误差均在 $3 \%$ 以内, 经过后验差检验的相对误差和均方根误 差也在 $5 \%$ 以内, 精度相比灰色预测方法有了很大提高。

从表 1 可知, 由于 2012 年 3 月份的用电量波动剧烈, 经 过平滑处理仍具有较大波动, 导致预测值有很大误差。本 文通过选取预测月份上一月、上一年度该月份及邻近月份 作为预测月份的最近邻居，即2013年2月、2012年2、3、4 月, 采用K最邻近算法对 2013 年 3 月用电量进行预测, 并与 粒子群算法的预测值进行组合优化, 有以下结果:

表5 2013年3月用电量的预测值。

\begin{tabular}{llll}
\hline & 灰色-粒子群 & K最邻近值法 & 组合优化 \\
\hline 预测值 & 767 & 685.9 & 699.5 \\
相对误差 (\%) & 14.65 & 2.53 & 4.36 \\
\hline
\end{tabular}


对 $K$ 最邻近值法及组合优化方法进行后验差检验, 并 与灰色-粒子群算法进行对比, 有以下结果:

表 6 对 3 月份数据预测的后验差检验。

\begin{tabular}{llll}
\hline & $\begin{array}{l}\text { 灰色-粒子 } \\
\text { 群 }\end{array}$ & $\begin{array}{l}\mathrm{K} \text { 最邻近值 } \\
\text { 法 }\end{array}$ & 组合优化 \\
\hline 平均相对误差 (\%) & 6.98 & 5.58 & 3.95 \\
均方根误差 (\%) & 8.55 & 6.42 & 4.33 \\
\hline
\end{tabular}

由表6可得, 经过 $K$ 最邻近算法的计算以及与粒子群算 法的优化组合, 在该算例中将预测方法的平均相对误差和 均方根误差准确度提高了近 $2 \%$ 。

\section{5. 结论}

本文通过分析灰色预测方程所存在的缺点, 通过粒子 群算法改进了灰色预测方程的求解过程, 建立了灰色一粒 子群预测模型, 实现了对月度用电量的准确预测, 通过与 $\mathrm{K}$ 最邻近算法的组合优化, 解决了原始数据波动太大导致 预测精度低的问题。主要结论如下:

（1）首先要对数据进行预处理, 避免因特殊原因导致 用电量激增或者锐减而无法准确预测下一年度该 月份的用电量, 合理的月度用电量数据是预测的 基础;

（2）通过粒子群算法对灰色预测方程的求解，通过迭 代方法对参数 $\mathrm{a}, \mathrm{b}$ 在选定区域内搜索最优解, 避 免了灰色预测方程中常数 $\mathrm{C}$ 所带来的误差, 消除了 因构造邻近均值序列和一阶微分方程导致的算法 误差, 提高了算法的准确度;

（3）通过与K最邻近算法的组合优化, 提高了算法的准 确度, 通过实例验证了本文所提出的方法在月度 用电量预测方面的有效性和准确性。

\section{参考文献}

[1] 康重庆, 夏清, 刘梅. 电力系统负荷预测 [M]. 北京: 中国电 力出版社, 2007 。

[2] 牛东晓, 曹树华, 等. 电力负荷预测技术及应用 [M]. 北京: 中国电力出版社, 1998。

[3] 吴安平. 产业电力弹性系数的意义及其在负荷预测中的应 用 $[J]$. 中国电力, 1998, 31 (12)：41-44。

[4] 毛玉风. 基于时间序列分析的电力需求预测及季节调整模 型的研究 [D]. 北京: 北京工业大学, 2013。
[5] 李瑾, 刘金朋, 王建军. 采用支持向量机和模拟退火算法的 中长期负荷预测方法 $[J]$. 中国电机工程学报, 2011,31(16): 63-66。

[6] 陆宁, 周建中, 何耀耀. 粒子群优化的神经网络模型在短期 负荷预测中的应用 [J]. 电力系统保护与控制, 2010, 38 (12): $65-68$ 。

[7] 唐慧. 基于混合模拟退火算法和SVM的电网协调性发展水平 评价研究 [J]. 江苏商论. 2010, (04) : 119-121。

[8] 牛东晓, 谷志红, 邢棉, 等. 基于数据挖掘的SVM短期负荷 预测方法研究 [J]. 中国电机工程学报, 2006, (18):6-12。

[9] Morita Hironobu, ZHANG Deping, Tamura Yasuo. Long-term load forecasting using gray system theory [J]. Electrical Engineering in Japan, 1995, $115(2)$ : $11-20$.

[10] Hsu CC, Chen CY. A modified grey forecasting model for long-term prediction $[\mathrm{J}]$. Journal of the Chinese institute of engineers. 2003, 26(3) : 301-308.

[11] WANG Shuohe, WAN Jianru, CHANG Yujian, et al. Medium-long term load forecasting based on improved grey model [C]. Proceedings of the Sixth international Conference on Machine Learning and Cybernetics, ICMLC 2007, 2007 (5) : 2520-2524.

[12] Feng G H, YANG Xiang, ZHANG Bingyi. The application of combined optimum grey model in medium and long term load forecasting [C]. 2006 China International Conference on Electricity Distribution, CICED. 2006: $51-55$.

[13] 谢宏, 魏江平, 刘鹤立. 短期负荷预测中支持向量机模型的 参数选取和优化方法 $[\mathrm{J}]$. 中国电机工程学报, 2009, 26 (22) : 17-22。

[14] 马洪松. 基于改进灰色模型的电力负荷预测方法研究及应 用 [D]. 保定: 华北电力大学, 2013。

[15] 叶猛. 基于灰色关联和回归分析的社会用电量预测研究 [J]. 价值工程, 2010，（36）：211-212。

[16] 牛勇, 王震宇, 王红军, 等. 改进灰色模型在中长期电力负 荷预测中的应用 $[\mathrm{J}]$. 东北电力大学学报, 2009, (02) :64-68。

[17] 皮薇, 黎特. 基于粗粘集改进的支持向量机模型及其在短期 电力负荷预测中的应用 $[\mathrm{J}]$. 山东电力高等专科学校学报, 2013，04: 16-21。

[18] 黎特, 皮薇. 支持向量机模型改进及在短期边际电价预测中 的应用 $[J]$. 国网技术学院学报, 2013，05：1-5。 